\title{
EL SENTIDO EN LA FUNCIÓN REFERENCIAL DE FRASES SUSTANTIVAS DEL ESPAÑOL
}

El registro de los significados léxicos y los mecanismos de su combinación, basados en el sistema de la lengua que todo hablante conoce inconscientemente, producen el sentido de las expresiones lingüísticas; mediante éste nos es posible, a los hablantes, relacionar cada expresión lingüística con su referente, en cada situación particular de uso.

Para que una expresión lingüística pueda referirse a algo, es necesario que mediante su sentido se delimiten sus posibilidades referenciales; al ámbito de estas posibilidades lo llamaré "extensión" ", para reservar el término "referente" a los casos particulares de uso, en los que intervienen los contextos situacionales. Utilizando de tal manera estos términos es posible describir el referente como el objeto del mundo (físico o imaginario) con el cual se relaciona una expresión mediante la interacción entre la extensión y la información del contexto (situacional y lingüístico).

En las frases sustantivas del español la delimitación de la extensión se realiza de dos maneras. Una de ellas se basa en alguna propiedad que el sentido de un modificador exige que cumplan los miembros de un conjunto, para formar parte del posible referente; mediante esta manera de delimitar la extensión pueden descartarse algunos miembros de un conjunto como posibles referentes, por no tener la propiedad en cuestión. La otra manera se ba-

1 Aunque actualmente sea usual emplear los términos "extensión" y "referencia"' como intercambiables, entre el significado, propio de una expresión, y el referente, propio del uso de la expresión, se encuentra algo: un conjunto de los objetos o situaciones que podrían ser los referentes de la expresión; a este conjunto lo llamo "extensión"; la particularización de uno de estos objetos o situaciones como referente depende de la interacción entre la extensión y el contexto de un uso particular. 
sa en los sentidos de los modificadores que no exigen que los miembros de un conjunto cumplan con alguna propiedad; mediante esta delimitación extensional no puede descartarse ningún miembro de un conjunto como posible referente ${ }^{2}$.

Cuando se combina el significado de un determinante con el significado de un sustantivo (o con el significado que se obtiene de la combinación de éste con sus otros modificadores, en una estructura mayor), en este último se produce una delimitación de la primera manera descrita, si el determinante es definido: en el sentido del sustantivo (o de la estructura formada por éste y modificadores) se delimitan las posibilidades referenciales ("delimitación extensional") mediante la exigencia de cumplir con una propiedad; si el determinante es indefinido, en el significado del sustantivo (o de la estructura correspondiente) se delimitan sus posibilidades referenciales ("delimitación extensional") sin la exigencia de cumplir con una propiedad; es decir, sin la posibilidad de descartar miembros como referentes potenciales.

Con el fin de ejemplificar la delimitación extensional mediante la exigencia de cumplir con una propiedad, trataré de representar, para algunos determinantes definidos, esa parte del sentido que resulta pertinente para tal función.

EL

$\longrightarrow$ El conjunto delimitado extensionalmente por los otros constituyentes de la frase sustantiva y que tiene la propiedad de pertenecer al universo del discurso ${ }^{3}$.

ESTE

$\longrightarrow$ Del conjunto delimitado extensionalmente por los otros constituyentes de la frase sustantiva, el subconjunto que

${ }^{2}$ En J. García FaJARdo, El sentido de los sintagmas nominales y los tipos de predicación, Instituto Nacional de Antropología e Historia, México, 1985 (en adelante $S S N y T P$ ), se mencionan las dos maneras de delimitación extensional que aquí se exponen.

${ }^{3}$ En el presente trabajo no analizo la delimitación del universo del discurso (en la cual interviene la delimitación extensional de la frase sustantiva); pero doy por hecho que cuando la delimitación contextual del universo del discurso no atañe al conjunto delimitado extensionalmente por los otros constituyentes de la frase sustantiva (es decir, por los constituyentes de dicha frase a excepción del determinante), este conjunto, al ser delimitado extensionalmente, queda incluido en dicho universo. 

de primera cercanía contextual ${ }^{4}$. mente por los otros constituyentes de la frase sustantiva, el subconjunto que está en relación directa con el hablante.

Con estos significados, los determinantes definidos delimitan la extensión de la frase sustantiva, con la posibilidad de deseartar, del conjunto delimitado por los otros constituyentes, los miembros que no cumplen con la propiedad aludida en cada caso:

EL LIBRO

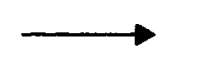

Del conjunto (universal) de libros, se descartan los que no pertenecen al universo del discurso (si queda más de uno, la referencia de la frase será la llamada tradicionalmente "genérica"; si la delimitación contextual del universo del discurso no atañe al conjunto de libros, el conjunto universal de tales pasará a incluirse en el discurso).

ESTE LIBRO

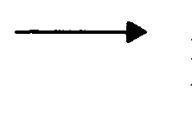

Del conjunto de libros, se descartan los que no están en relación de primera cercanía contextual (si queda más de uno, la referencia será "genérica", como en el caso de una plática sobre los libros de una editorial, que se orientara a hablar de los libros que menos se venden y, una vez que se hubiera hecho referencia a los libros científicos, se dijese: "este libro tiene un público muy reducido"').

4 La relación de cercanía contextual puede establecerse en las dimensiones del texto o del contexto extralingüístico (espacial o temporal); en el primer caso, se tratará del último subconjunto mencionado, incluido en el conjunto delimitado por los otros constituyentes de la frase sustantiva; en el segundo caso, se referirá al subconjunto más cercano en el espacio al lugar de la enunciación, o al más cercano en el tiempo al momento de la enunciación. Por supuesto, los distintos grados de cercanía no son absolutos sino relativos entre ellos mismos. 
MI LIBRO

Del conjunto de libros, se descartan los que no están en relación directa con el hablante (si queda más de uno, la referencia será "genérica", tal y como resultaría si en el discurso descrito para ejemplificar la referencia genérica de este libro, el director de la editorial, a continuación, dijese: "sin embargo, mi libro tiene tal nivel de calidad y una distribución tan bien planificada, que goza de gran demanda en todos sus géneros").

Como ejemplos de delimitación extensional sin descarte de miembros, entre los determinantes, función propia de los indefinidos, podemos considerar los siguientes:

UN

$\longrightarrow$ Del conjunto delimitado extensionalmente por los otros constituyentes de la frase sustantiva, un solo miembro ${ }^{5}$, o todos los miembros (en cuyo caso la referencia será "genérica"; como en expresiones del tipo: "un perro tiene cuatro patas').

UNOS

$\longrightarrow$ Del conjunto delimitado por los otros constituyentes de la frase sustantiva, un subconjunto formado por varios miembros.

CUATRO

Del conjunto delimitado extensionalmente por los otros constituyentes de

${ }^{5}$ La exigencia de la propiedad de pertenecer al universo del discurso, que corresponde al determinante definido $e l$, contrasta claramente con la ausencia de tal en el determinante indefinido un, cuando ambos toman como referencia un solo miembro (que, en el caso del primer determinante, equivaldría al conjunto unimembre del universo del discurso): ocasiona extrañeza una expresión como "entró el joven a la tienda de la esquina", cuando en el universo del discurso no está incluido un conjunto (unimembre) de jóvenes (extrañeza que me parece se maneja, a veces, en el discurso literario); mientras que, en las mismas circunstancias, no causa extrañeza una expresión como "entró un joven a la tienda de la esquina". 
la frase sustantiva, un subconjunto formado por cuatro miembros.

TODO

$\longrightarrow$ Del conjunto delimitado extensionalmente por los otros constituyentes de la frase sustantiva, cada uno de sus miembros.

En las representaciones de los sentidos de los determinantes indefinidos que se ofrecen arriba, puede apreciarse la ausencia de restricción de la extensión en cuanto a la función de descartar miembros como posibles referentes, a través de la exigencia de cumplir con una propiedad. Por ejemplo, en "compré un libro", un expresa que se hace referencia a uno de los miembros del conljunto de libros; pero, al delimitar la extensión de este conjunto a un solo miembro, no se descarta a ningún individuo como posible referente (mientras sea un miembro de dicho conjunto, puede pertenecer o no al universo del discurso, mantener cualquier nivel en la relación de cercanía contextual y en la relación con el hablante, etcétera).

Por otra parte, al combinar el significado del núcleo de la frase sustantiva con el significado de un adjetivo, la delimitación de la extensión depende de dos factores; el primero es el lugar que ocupa el adjetivo con respecto al sustantivo ${ }^{6}$; el segundo es el tipo de determinante de la frase.

Atendiendo al primer factor, podemos decir que el adjetivo delimita la extensión de la frase con descarte de miembros (mediante la exigencia de que éstos cumplan con alguna propiedad: la que se menciona mediante el adjetivo), únicamente en el caso de que el adjetivo esté pospuesto al sustantivo; esta forma de delimitar la extensión es propia, no sólo de los adjetivos léxicos pospuestos, sino de cualquier estructura pospuesta al sustantivo que sea modificadora de éste, a excepción de las oraciones relativas explicativas y, en general, de las aposiciones explicativas, que funcionan semánticamente como los adjetivos antepuestos, sólo en parte ${ }^{7}$. Cuando el adjetivo antecede al sustantivo, no funciona co-

${ }^{6}$ En $S S N y T P$ se describe la combinación del significado del adjetivo con el significado del sustantivo, mediante los tipos de predicación, según aquél esté antepuesto o pospuesto a éste y de acuerdo, también, con el tipo de determinante.

${ }^{7}$ Las oraciones adjetivas explicativas (o "relativas explicativas") y, en ge- 
mo delimitador de la extensión: la propiedad que se menciona mediante el adjetivo, en este caso, no se relaciona con los miembros del conjunto al que se refiere el sustantivo, descartando a los que no cumplan con ella, sino que se relaciona con el conjunto, como totalidad, expresando la presuposición de que -en el universo del discurso - el conjunto entero al que se refiere el sustantivo tiene la propiedad en cuestión; y al relacionarse con el conjunto entero que corresponde a la extensión del sustantivo, no delimita dicha extensión. Contrástense:

Los espectadores exigentes aplaudieron poco Los exigentes espectadores aplaudieron poco

en donde exigentes, al estar pospuesto al sustantivo, funciona como delimitador de la extensión de éste, mientras que al antecederlo no funciona como tal, sino que origina la presuposición de que todos los miembros del conjunto del sustantivo tienen la propiedad de ser exigentes.

Ahora bien, en cuanto al tipo de determinante de la frase, lo que resulta pertinente en la delimitación de la extensión mediante el adjetivo es que el determinante genere el sentido de conjunto o bien que genere el sentido individual en la frase ${ }^{8}$. Los determinantes definidos ( $y$ los indefinidos en plural), al originar el sentido de conjunto, permitirán la relación del significado del adjetivo antepuesto con el conjunto entero de la extensión del sustantivo, produciendo en la frase un significado que expresa la presu-

neral, las aposiciones explicativas, semánticamente tienen en común con los adjetivos antepuestos al sustantivo la característica de no delimitar la extensión de la frase de la que son constituyentes, sino que se relacionan con la totalidad del conjunto que corresponde a la extensión del sustantivo (o del sustantivo más otros modificadores), expresando que -en el universo del discursoel conjunto entero al que se refiere el sustantivo (o la cadena correspondiente) tiene la propiedad a la que se refiere la explicativa. La diferencia semántica entre estas estructuras explicativas pospuestas y los adjetivos antepuestos consiste en que estos últimos expresan una presuposición, mientras que las explicativas pospuestas expresan una aseveración semejante a la que expresan las oraciones independientes. El tema de esta diferencia se tratará en un trabajo posterior.

${ }^{8}$ En J. García Fajardo, Un estudio del significado (presupuestos, principios y desarrollo), Instituto Nacional de Antropología e Historia, México (en prensa), se muestra que los determinantes definidos, la marca de plural y la conjunción de frases generan el sentido de conjunto; mientras que los determinantes indefinidos generan el sentido individual. 
posición de que el conjunto en su totalidad tiene - en el universo del discurso - la propiedad aludida por el adjetivo. Contrástese:

Los honorables colegas serán invitados

de donde surge la presuposición de que todos los miembros del conjunto de colegas son honorables, frente a:

Los colegas honorables serán invitados

donde " honorables" delimita la extensión del conjunto de colegas.

Los determinantes indefinidos en singular, al originar el sentido individual en la frase no permitirán la relación del significado del adjetivo antepuesto con el conjunto entero de la extensión. Por consiguiente, si el determinante es indefinido (y la frase está en singular), aunque el adjetivo esté antepuesto, el significado de éste funcionará como delimitador de la extensión de la frase -igual que si estuviera pospuesto- descartando, del conjunto que constituye la extensión del sustantivo, a los miembros que no cumplan con la propiedad mencionada en el adjetivo, por lo que entre los siguientes ejemplos no se percibe un cambio en la delimitación de la extensión:

Todo espectador exigente aplaudió poco

Todo exigente espectador aplaudió poco

Resumiendo, la delimitación de la extensión (proceso necesario para que las expresiones tengan una función referencial) de las frases sustantivas se lleva a cabo en dos formas distintas; cada una de ellas depende de la categoría sintáctica - determinảnte definido y adjetivos/determinante indefinido - que se combina con el sustantivo y de su posición con respecto a éste - para los adjetivos: propuesto/antepuesto-; además, hemos observado que la forma de combinación del adjetivo antepuesto depende del sentido que se origina en el determinante (de conjunto o individual). La extensión, delimitada mediante estos factores, permite relacionar cada frase sustantiva con su referente.

En otras palabras, podemos decir que el sentido del núcleo de la frase sustantiva delimita la extensión de éste; podemos imaginar dicha extensión como un conjunto (el conjunto universal de miembros que tienen la propiedad mencionada en el sustantivo). El sentido del adjetivo selecciona, de ese conjunto, a los miem- 
bros que tienen la propiedad mencionada en el adjetivo, delimitando la extensión de la estructura a un subconjunto del conjunto original; esto, en caso de que el adjetivo esté pospuesto al sustantivo. Si el adjetivo está antepuesto, no se produce un proceso de delimitación de la extensión, pues el sentido del adjetivo se relaciona, en este caso, con el conjunto entero del sustantivo - no con cada miembro- originando la presuposición de que todos los miembros del conjunto mencionado por el sustantivo tienen la propiedad mencionada en el adjetivo; esto ocurre siempre que el adjetivo antecede al sustantivo, a menos de que el determinante de la frase genere en ésta el sentido individual (determinante indefinido), en cuyo caso no podrá relacionarse el sentido del adjetivo con el conjunto entero del sustantivo, sino con los miembros de éste, ocasionando el proceso de delimitación de la extensión, por selección de miembros, como en el caso del adjetivo pospuesto.

El sentido del determinante, además de definir la forma como se combina el significado del adjetivo con el significado del sustantivo, delimita la extensión de toda la frase de dos maneras distintas, dependiendo cada una de la clase de determinante de que se trate. Si éste es indefinido, reduce cuantitativamente la extensión del conjunto a un subconjunto: sin seleccionar sus miembros mediante la exigencia de alguna propiedad. Si es definido, en cambio, delimita la extensión del conjunto obtenido mediante la combinación del sentido del sustantivo y del adjetivo, seleccionando en aquél los miembros que tengan la propiedad exigida por el significado del determinante.

Una vez delimitada la extensión de la frase, por procesos originados en el sistema mismo de la lengua, al interactuar la extensión con la información del contexto, en el uso de la expresión, se llega al referente de la frase. 